\title{
trans-Selective and Switchable Arene Hydrogenation of Phenol Derivatives
}

\author{
Marco Wollenburg, ${ }^{\ddagger}$ Arne Heusler, ${ }^{\ddagger}$ Klaus Bergander, and Frank Glorius* \\ Cite This: ACS Catal. 2020, 10, 11365-11370 \\ Read Online
}

ABSTRACT: A trans-selective arene hydrogenation of abundant phenol derivatives catalyzed by a commercially available heterogeneous palladium catalyst is reported. The described method tolerates a variety of functional groups and provides access to a broad scope of trans-configurated cyclohexanols as potential building blocks for life sciences and beyond in a one-step procedure. The transformation is strategically important because arene hydrogenation preferentially

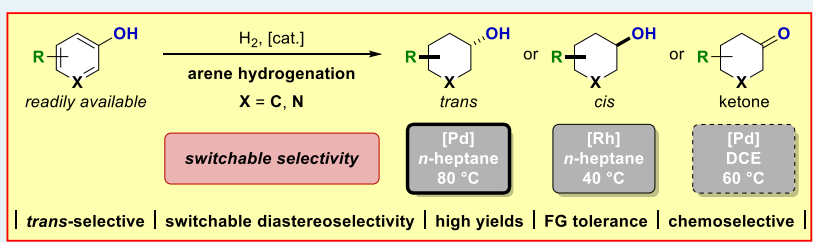
delivers the opposite cis-isomers. The diastereoselectivity of the phenol hydrogenation can be switched to the cis-isomers by employing rhodium-based catalysts. Moreover, a protocol for the chemoselective hydrogenation of phenols to cyclohexanones was developed.

KEYWORDS: arene hydrogenation, trans-selective, switchability, cyclohexanols, palladium catalysis

A rene hydrogenation is a powerful tool to transform simple, two-dimensional precursors into more complex, three-dimensional scaffolds. ${ }^{1}$ A plethora of readily available arenes and heteroarenes provided by established transformations (e.g., cross-coupling, aromatic substitution), offer potential access to a wide array of cyclic saturated motifs. The strategic importance of arene hydrogenation is exemplified by the industrial synthesis of cyclohexane ${ }^{2}$ and cyclohexene ${ }^{3}$ from benzene on multi-ton scale.

The field of arene hydrogenation has constantly evolved over the last several decades, and progress toward the stereoand chemoselective hydrogenation of (hetero)aromatic compounds has been achieved. Chemoselective hydrogenation of arenes provides direct access to saturated carbo- and heterocycles containing reductively labile functional groups directly attached to the reactive center. ${ }^{1 \mathrm{~d}, 4}$ The competing hydrodefunctionalization pathway observed for fluorinated, ${ }^{4 a-c}$ borylated $^{4 \mathrm{~d}, \mathrm{e}}$ or silylated ${ }^{4 \mathrm{f}}$ arenes can be limited by the choice of suitable reaction conditions. Additionally, the enantioselective hydrogenation of heteroarenes provides rapid access to chiral, saturated heterocycles through the controlled formation of stereocenters. ${ }^{5}$

During the (chemoselective) hydrogenation of multisubstituted aromatic compounds, multiple stereocenters are formed in one step allowing for the formation of several product diastereomers. In general, the transition-metal catalyzed hydrogenation of arenes favors the corresponding all-cis configuration of the saturated analogues (Scheme 1a, path I). ${ }^{1,4}$ The cis-selectivity results from a fast, continuous hydrogenation of the substrate through a non-interrupted coordination to the catalyst. The corresponding trans-isomers require a $\pi$-facial exchange of the dearomatized diene or olefin intermediate via substrate desorption and readsorption to the
Scheme 1. Diastereoselectivity in the Process of Arene Hydrogenation and Switchable (Stereo)selectivity in the Hydrogenation of Phenols

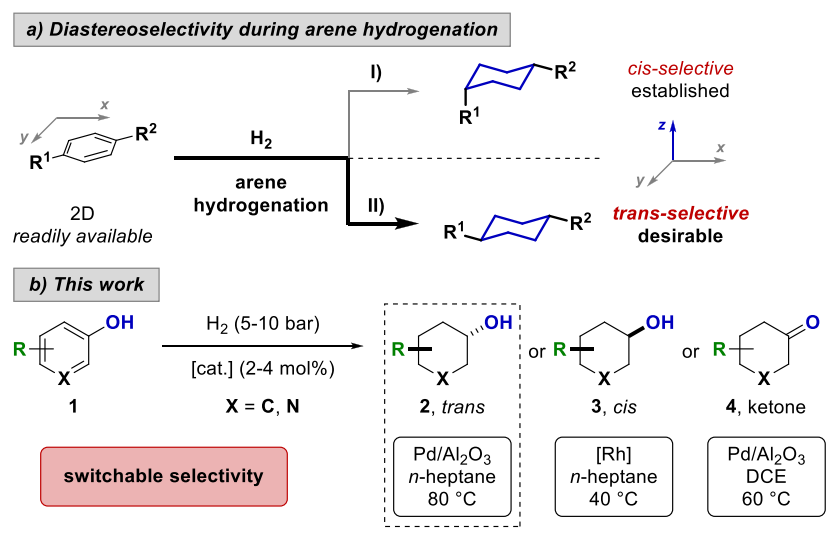

catalyst, which is associated with binding to the sterically more hindered $\pi$-face. For this reason, the corresponding transisomers remain minor side products during arene hydrogenation. ${ }^{1,4}$ However, trans-isomers of multisubstituted saturated carbo- and heterocycles are desirable product motifs, and their synthesis starting from the corresponding cis-isomers is

Received: August 5, 2020

Revised: September 9, 2020

Published: September 15, 2020 
often limited, rendering a direct trans-selective hydrogenation of arenes desirable (Scheme 1a, path II).

Encouraged by a few rare examples with diminished cisselectivity during our previous studies on the rhodium cyclic (alkyl)(amino)carbene ( $\mathrm{Rh}-\mathrm{CAAC}$ )-catalyzed chemoselective hydrogenation of arenes, ${ }^{4}$ we sought to investigate the underexplored and limited trans-selective hydrogenation of arenes..$^{6}$ Especially unprotected phenols gave a relatively high ratio of the "undesired" trans-diastereomer in our previous rhodium-catalyzed studies. The hydrogenation of phenol is an important industrial process for the synthesis of cyclohexanone and cyclohexanols as the intermediates for Nylon-6 and $66 .^{2,7}$ Substituted cyclohexanols are also extensively used as valuable chemical feedstock and synthetic intermediates in the pharmaceutical, petrochemical, and fine chemical industry. Moreover, the resulting hydroxyl group provides a diverse synthetic handle for further functionalization of the products.

Herein, we report the first comprehensive study toward a trans-selective hydrogenation of arenes. trans-Cyclohexanols are synthesized from abundant phenols in a one-step reaction that tolerates a variety of functional groups (Scheme $1 b$ ).

We commenced our studies by investigating different transition-metal based catalysts in the hydrogenation of $p$ cresol. When switching from rhodium-based catalysts to heterogeneous palladium catalysts, we noticed an inversion in the diastereoselectivity of the reaction. Investigating the efficacy of different palladium catalysts in the reaction revealed that a 5 wt $\% \mathrm{Pd} / \mathrm{Al}_{2} \mathrm{O}_{3}$ system had the best selectivity toward the desired trans-cyclohexanol product, accompanied by only trace amounts of the unwanted cyclohexanone intermediate. After optimization, employing commercially available $5 \mathrm{wt} \%$ $\mathrm{Pd} / \mathrm{Al}_{2} \mathrm{O}_{3}$ in $n$-heptane as the solvent, under a low hydrogen pressure of 5 bar at $80{ }^{\circ} \mathrm{C}$ provided the best transdiastereoselectivity, yield, and chemoselectivity of the reaction. Hydrogenation of $p$-cresol furnished 4-methylcyclohexanol 2a in 90\% yield and 80:20 diastereomeric ratio (d.r., trans:cis). ${ }^{8}$ To evaluate the sensitivity of our developed protocol, we conducted a reaction-condition-based sensitivity screen. ${ }^{9}$ The influence on yield and diastereoselectivity was investigated by systematic variation of key reaction parameters (see Supporting Information).

With the optimized conditions in hand, we began investigating the scope of the reaction (Scheme 2). The hydrogenation of phenols bearing aliphatic substituents (2ae) provided the corresponding trans-cyclohexanols in high yields. With increasing steric bulk in para-position, the diastereomeric ratio increased from 80:20 d.r. to 91:9 d.r. In addition, 4-tert-butylcyclohexanol $\mathbf{2 d}$ was obtained in quantitative yield and $88: 12$ d.r. after scale-up to $5.0 \mathrm{~g}$ scale ( 33.3 $\mathrm{mmol})$. Boc-protected $p$-aminophenol was hydrogenated to protected 4-aminocyclohexanol (2f) in 84\% yield and 80:20 d.r. By switching the solvent from $n$-heptane to polar isopropanol and adding $\mathrm{K}_{2} \mathrm{CO}_{3}$ as base, the unprotected $p$ aminophenol could be hydrogenated to $2 \mathrm{f}$ in high yield and with an increased diastereoselectivity of $88: 12$. Boc-protected trans-4-aminocyclohexanol could also be obtained after hydrogenation and protection of $p$-nitrophenol while reducing the nitro group and arene in a one-step procedure. Moreover, different ester substituents were tolerated furnishing transcyclohexanols with protected acids $(\mathbf{2} \mathbf{h}-\mathbf{j})$. Aryl boronic acid derivative $1 \mathbf{k}$ was hydrogenated giving access to the synthetically valuable trans-configurated organoboron compound $\mathbf{2 k}$. Additionally the organosilicon analogue 21 could be obtained
Scheme 2. Substrate Scope for the trans-Selective Hydrogenation of Phenols ${ }^{a}$

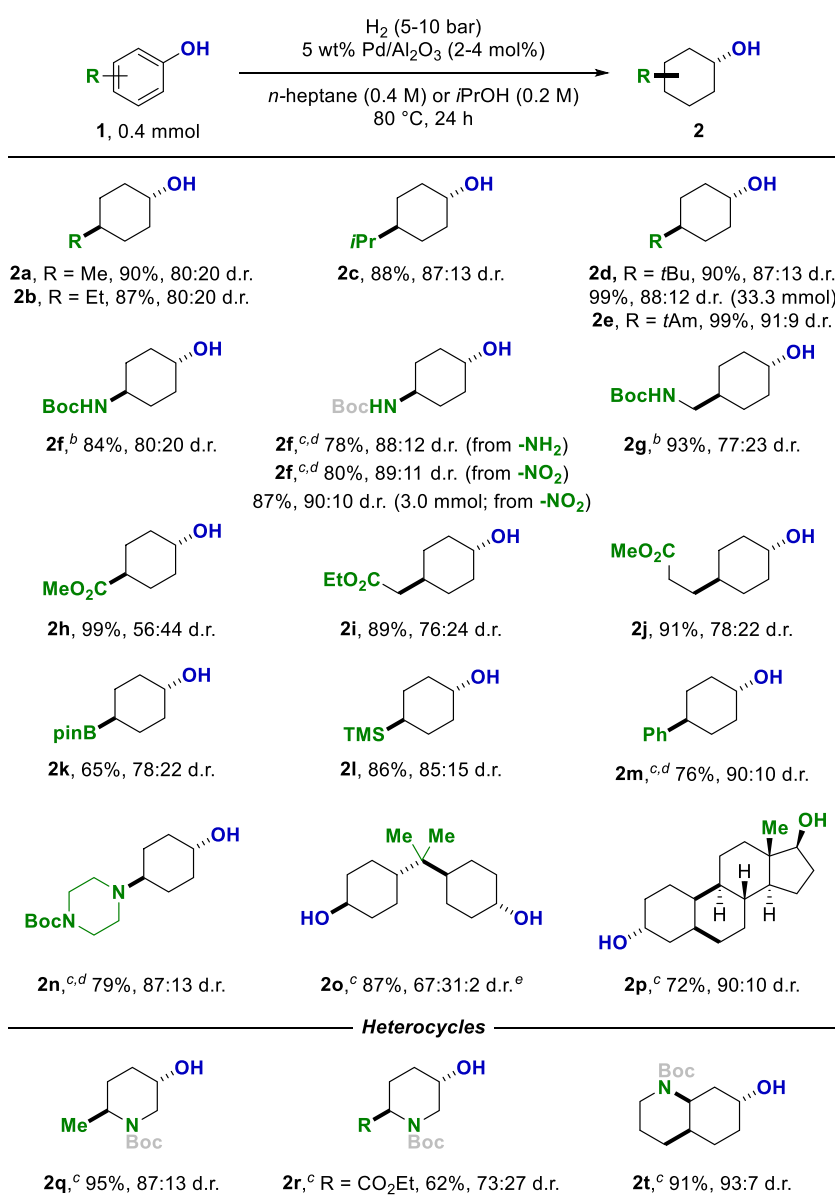

$\begin{aligned} \text { 2q, }{ }^{c} 95 \%, 87: 13 \text { d.r. } & \text { 2r, },{ }^{c} \mathrm{R}=\mathrm{CO}_{2} \mathrm{Et}, 62 \%, 73: 27 \text { d.r. } \\ 95 \%, 86: 14 \text { d.r. }(3.0 \mathrm{mmol}) & \mathbf{2 s},{ }^{c} \mathrm{R}=\mathrm{Ph}, 75 \%, 67: 33 \text { d.r. }\end{aligned}$

${ }^{a}$ Combined yields of isolated product after column chromatography are given. The d.r. values were determined by GC-MS or ${ }^{1} \mathrm{H}$ NMR analysis prior to purification. Piperidines and amines were trapped with $\mathrm{Boc}_{2} \mathrm{O}$ prior to isolation. For details, see Supporting Information. ${ }^{b} 48$ h. ${ }^{c} i \mathrm{PrOH}$ as solvent. ${ }^{d} \mathrm{~K}_{2} \mathrm{CO}_{3}$ as additive. ${ }^{e}$ The ratio is trans/ trans:trans/cis:cis/cis.

in $86 \%$ yield and $85: 15$ d.r. Chemoselective hydrogenation of the more substituted phenol ring compared to the phenyl substituent in $p$-phenylphenol delivered $2 \mathrm{~m}$ in $76 \%$ yield and 90:10 d.r. Additionally, a Boc-protected piperazine substituent was tolerated, giving the trans-configurated building block 2 n with two orthogonal sites available for further functionalization. The hydrogenation of bisphenol A, a common precursor for polycarbonates, provided compound 20 bearing two transconfigurated cyclohexanols as the major isomer. Showcased by products 2a-2o, a wide array of achiral building blocks with potential applications in pharmaceutical sciences could be synthesized. Moreover, the female sex hormone estradiol could be transformed into the corresponding saturated analogue $2 p$ in $72 \%$ combined yield and 90:10 d.r.

Since saturated heterocycles are an important structural motif, we extended the scope of the trans-selective hydrogenation to different heteroarenes $(\mathbf{2 q - t})$. By switching to isopropanol as polar solvent, poisoning of the palladium catalyst could be prevented. ${ }^{10,1 h}$ Hydroxypyridine $\mathbf{1 q}$ was hydrogenated to the corresponding piperidine in $95 \%$ yield and 87:13 d.r. The reactivity and selectivity were preserved 
after scale-up of the developed method to $3 \mathrm{mmol}$. Reductively labile groups like esters (2r) and phenyl substituents (2s) were tolerated, enabling a trans- and chemoselective hydrogenation of pyridines. Additionally, quinoline derivative 1t was transselectively hydrogenated, furnishing the perhydro product in 91\% yield and 93:7 diastereomeric ratio. In almost all cases, the major diastereomer could be separated by column chromatography, providing access to the diastereomerically pure transconfigurated (heterocyclic) cyclohexanol derivatives with a broad variety of functional groups. The trans-selectivity of the hydrogenation was confirmed by X-ray diffraction analysis of products $\mathbf{2 f}, \mathbf{2 m}, \mathbf{2 o}$, and $\mathbf{2 q}$. Furthermore, the configuration of the majority of products were confirmed by NMR analysis. ${ }^{8}$

In many cases both product diastereomers of a newly developed method are of synthetic interest. However, switching the diastereoselectivity of established and newly developed protocols often remains non-trivial, and long synthetic routes are necessary. The cis-selective hydrogenation of phenols is frequently limited by poor functional group tolerance and cleavage of the carbon-oxygen bond by hydrogenolysis when employing standard heterogeneous catalysts (e.g., $\mathrm{Rh} / \mathrm{C}, \mathrm{Rh} / \mathrm{Al}_{2} \mathrm{O}_{3}$ ) or $[\mathrm{Rh}(\mathrm{COD}) \mathrm{Cl}]_{2}$ in combination with various catalyst supports. ${ }^{11,6 \mathrm{~d}}$ When using $\mathrm{Rh}-\mathrm{CAAC}$ as a precatalyst ${ }^{12}$ and $4 \AA$ molecular sieves or silica gel as catalyst support, we could switch the diastereoselectivity to the corresponding cis-diastereomers without the undesired hydrogenolysis (Scheme 3).

Scheme 3. Substrate Scope for the cis-Selective Hydrogenation of Phenols ${ }^{a}$

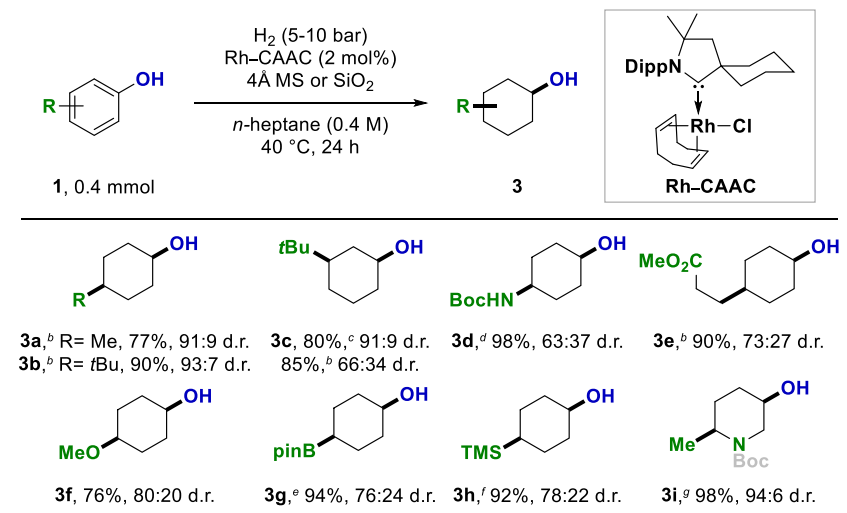

${ }^{a}$ Combined yields of isolated product after column chromatography are given. The d.r. values were determined by GC-MS or ${ }^{1} \mathrm{H}$ NMR analysis prior to purification. Piperidines were trapped with $\mathrm{Boc}_{2} \mathrm{O}$ prior to isolation. For details, see Supporting Information. CAAC = cyclic (alkyl) (amino)carbene. Dipp = 2,6-diisopropylphenyl. ${ }^{b}[\mathrm{Rh}$ (COD)Cl $]_{2}(2 \mathrm{~mol} \%) .{ }^{c} 5 \mathrm{wt} \% \mathrm{Pd} / \mathrm{Al}_{2} \mathrm{O}_{3}(4 \mathrm{~mol} \%) .{ }^{d} 48 \mathrm{~h} .{ }^{e} \mathrm{H}_{2}(50$ bar). ${ }_{\mathrm{H}_{2}}(20 \mathrm{bar}) .{ }^{g_{i} \mathrm{PrOH} \text { as solvent. }}$

Phenols with simple alkyl chains could be hydrogenated using $[\mathrm{Rh}(\mathrm{COD}) \mathrm{Cl}]_{2}$ as a catalyst. The hydrogenation of $p$ cresol and $p$-tert-butylphenol provided the corresponding cyclohexanols $\mathbf{3 a}$ and $\mathbf{3 b}$ in high yields and diastereoselectivities. $m$-tert-Butylphenol was hydrogenated and 3-tert-butylcyclohexanol 3c was obtained in $85 \%$ yield and 66:34 d.r. (cis:trans) when using $[\mathrm{Rh}(\mathrm{COD}) \mathrm{Cl}]_{2}$ as a catalyst. The diastereoselectivity of the reaction could be increased to 91:9 d.r. when using palladium on alumina instead. For phenols decorated with more labile functional groups, the $\mathrm{Rh}-\mathrm{CAAC}$ system was used. Boc-protected cis-4-aminocyclohexanol $\mathbf{3 d}$ and cyclohexanol $3 \mathbf{e}$ with an ester substituent were obtained in excellent yields. Moreover, ether (3f), organoboron (3g), and organosilicon (3h) functionalities were well tolerated, rendering cis-configurated cyclohexanols with various functional groups accessible. The cis-selective hydrogenation protocol could be extended to pyridines, demonstrated by the hydrogenation of hydroxypyridine $2 \mathbf{i}$. When using $[\mathrm{Rh}(\mathrm{COD}) \mathrm{Cl}]_{2}$ as the catalyst undesired hydrogenolysis of functional groups was observed. For example, cleavage of the carbon-oxygen bond ( $3 \mathbf{f})$, carbon-silicon bond ( $3 \mathbf{h})$, and hydrodeborylation $(\mathbf{3 g})$ could be detected (see Supporting Information for more details).

The chemoselective hydrogenation of phenols to cyclohexanones has been focus of different studies. ${ }^{13,4 \mathrm{~g}}$ The major challenge associated with chemoselective phenol hydrogenation is preventing the over-reduction of the reductively more labile carbonyl group. Herein, we present a simple variation of our protocol by using the same, commercially available 5 wt \% $\mathrm{Pd} / \mathrm{Al}_{2} \mathrm{O}_{3}$ as catalyst, 5 bar of hydrogen, and 1,2-dichloroethane (DCE) as solvent to target this challenge (Scheme 4). Hydrogenation of $p$-cresol and o-tert-butylphenol in DCE provided 4-methylcyclohexanone $4 \mathrm{a}$ in $81 \%$ yield and 2-tertbutylcyclohexanone $4 \mathrm{~b}$ in $85 \%$ yield.

Scheme 4. Substrate Scope for the Ketone-Selective Hydrogenation of Phenols ${ }^{a}$

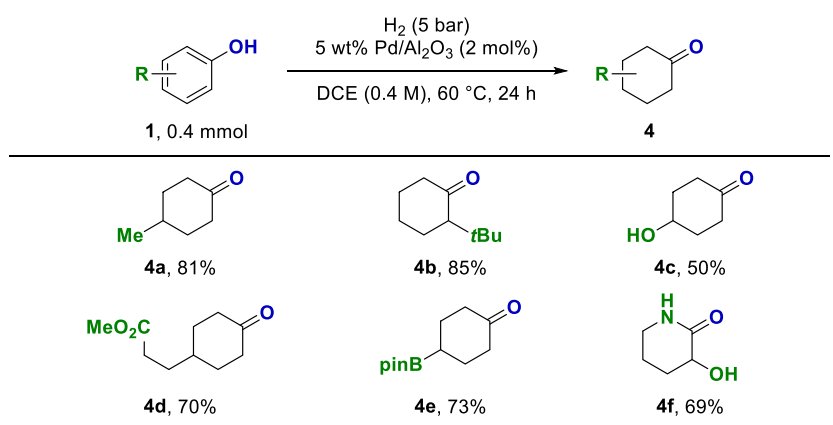

${ }^{a}$ Yields of isolated product after column chromatography are given. DCE $=1,2$-dichloroethane.

During the hydrogenation of hydroquinone, reduction of one carbonyl group of the diketo intermediate was observed, and 4-hydroxycyclohexanone $4 \mathrm{c}$ was obtained as the major product in $50 \%$ yield. Reductively labile groups like esters (4d) and pinacol boronic esters (4e) were tolerated, and the cyclohexanone derivatives were obtained in $70 \%$ and $73 \%$ yield, respectively. The major side products during the chemoselective hydrogenation of phenols to cyclohexanones were the corresponding cyclohexanols (see Supporting Information).

Having established three valuable protocols for the diastereo- and chemoselective hydrogenation of phenols, we sought to investigate the influence of the substitution pattern on reactivity and diastereoselectivity (Scheme 5). During the palladium-catalyzed hydrogenation of $p$-cresol, the thermodynamically more stable trans-4-methylcyclohexanol 2a was obtained as the major diastereomer. When employing a rhodium-based catalyst, the diastereoselectivity was inverted to the thermodynamically less stable cis-4-methylcyclohexanol 3a. When changing the substrate from $p$-cresol to $m$-cresol, cis-3methylcyclohexanol $3 \mathrm{ab}$ was obtained as the major product for both catalytic systems ( $\mathrm{Pd}$ and $\mathrm{Rh}$ ). The same selectivity was 
Scheme 5. Substitution Pattern and Diastereoselectivity for the Hydrogenation of Phenols ${ }^{a}$

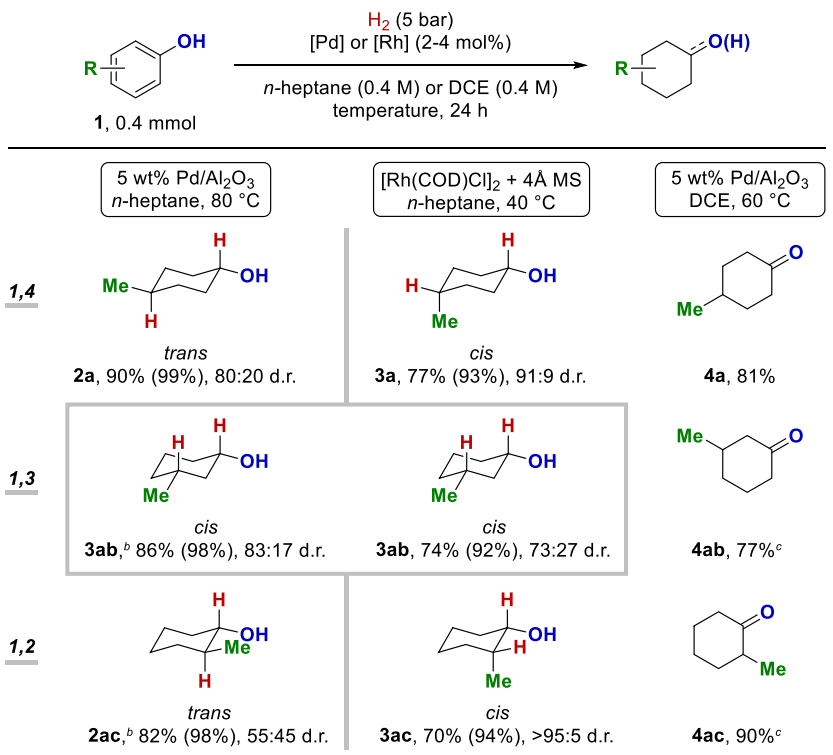

${ }^{a}$ Combined yields of isolated product after column chromatography are given. Diastereoselectivity and yields in parentheses were determined by ${ }^{1} \mathrm{H}$ NMR analysis. For details, see Supporting Information. ${ }^{b} \mathrm{H}_{2}$ (10 bar). ${ }^{c}$ Yield determined by GC-FID analysis.

previously observed in the hydrogenation of $m$-tert-butylphenol (Scheme 3). The use of palladium on alumina as the catalyst furnished a higher ratio of the thermodynamically more stable cis-diastereomer in the case of 1,3-disubsituted cycloalkanes. The palladium-catalyzed hydrogenation of $o$-cresol provided trans-2-cyclohexanol 2ac with low diastereoselectivity (55:45 d.r.), although trans-1,2-disubstituted cycloalkanes are thermodynamically favored. When employing the rhodium-based catalytic system, an excellent diastereoselectivity of $>95: 5$ d.r. for cis-2-methylcyclohexanol 3ac was observed. The chemoselective hydrogenation of phenols to cyclohexanones showed no significant influence caused by the substitution pattern, giving access to the volatile methylcyclohexanones $4 a-4 a c$ in good yields. The catalytic system based on rhodium provides products generated by the all-cis addition ${ }^{1,4}$ of hydrogen atoms to the substrates, whereas the catalytic system based on palladium gives access to the thermodynamically more stable diastereomers.

The proposed mechanism for the heterogeneous hydrogenation of phenols to cyclohexanols involves partially hydrogenated cyclohexenols (enol-forms) and the corresponding cyclohexanones (keto-enol tautomerism). ${ }^{14}$ The transisomers are believed to be formed through desorption and readsorption of intermediate cyclohexanone/cyclohexenol species ( $\pi$-facial exchange) followed by the cis-addition of hydrogen to enol intermediates. ${ }^{1 \mathrm{~d}, 15}$

Mechanistic experiments showed a fast consumption of the starting material and formation of 4-tert-butylcyclohexanone as an intermediate (see Supporting Information for more details). However, direct hydrogenation of 4-tert-butylcyclohexanone resulted in reduced diastereoselectivity (67:33 d.r.), compared with the hydrogenation of p-tert-butylphenol (87:13 d.r.). Hydrogenation of "diene" intermediates (e.g., 4-tert-butylcyclohex-2-en-1-one and 4-tert-butylcyclohex-3-en-1-one) resulted in roughly the same diastereoselectivity as the direct hydrogenation of $p$-tert-butylphenol. Diene intermediates could never be observed because of the fast hydrogenation of alkene double bonds. Key to the increased trans-selectivity during phenol hydrogenation could be the low concentration of the cyclohexanone intermediate, which disfavors the slow, direct ketone hydrogenation, forming the minor cis-isomer. The trans-isomer could be formed through a desorption and readsorption process of diene and enol intermediates, which is facilitated through keto-enol tautomerism. Deuteration studies show deuterium scrambling at the 2-position, supporting the rapid interconversion of keto and enol intermediates on the catalyst surface. In addition, increasing steric bulk in 4-position as well as the high reaction temperature favor the process of desorption and readsorption ( $\pi$-facial exchange) and therefore increase the ratio of transproducts. Moreover, diastereomerically pure trans- and cis-4tert-butylcyclohexanols (>99:1 d.r.) were subjected to the standard conditions, and no isomerization was observed, excluding a thermodynamically driven isomerization process.

Having established an efficient and versatile method for the trans-selective hydrogenation of phenols, we sought to demonstrate the synthetic utility of the obtained products (Scheme 6). Acetylation of trans-4-tert-butylcyclohexanol 2d

Scheme 6. Synthetic Applications of trans-Cyclohexanols ${ }^{a}$

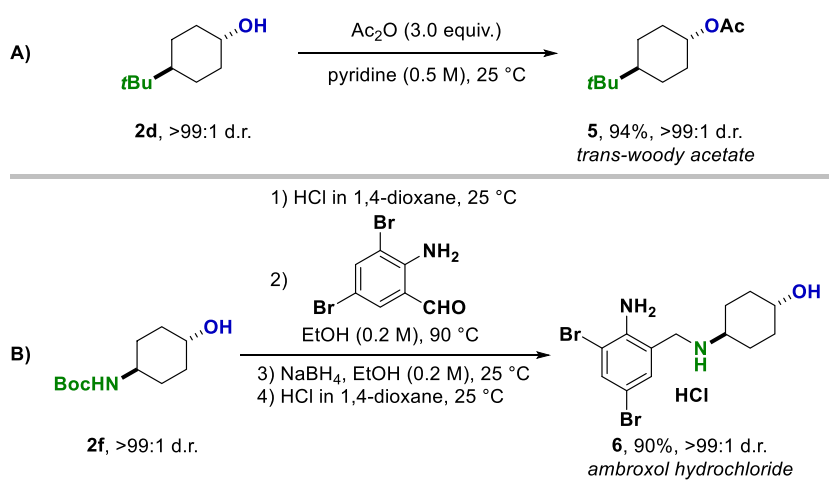

${ }^{a}$ For experimental details, see Supporting Information.

provided the trans-isomer of woody acetate 5, a fragrance ingredient used in cosmetics. ${ }^{16}$ Furthermore, ambroxol, a mucolytic agent used in the treatment of respiratory diseases, was synthesized. ${ }^{17}$ Starting from inexpensive $p$-nitrophenol, Boc-protected trans-4-aminocyclohexanol $2 \mathrm{f}$ was obtained in $87 \%$ yield and 90:10 d.r. on a $3 \mathrm{mmol}$ scale in one step (Scheme 2). After separation of the diastereomers on silica gel, treatment of $2 \mathrm{f}$ with $\mathrm{HCl}$ in 1,4-dioxane provided diastereomerically pure 4-trans-aminocyclohexanol. Imine formation with 2-amino-3,5-dibromobenzaldehyde, followed by $\mathrm{NaBH}_{4}$ reduction and treatment with $\mathrm{HCl}$ gave access to ambroxol hydrochloride in $90 \%$ yield over four steps (Scheme 6B).

In conclusion, we have developed three sets of reaction conditions for the hydrogenation of abundant phenols providing access to either trans- or cis-configurated cyclohexanols, as well as cyclohexanones. The trans-selective hydrogenation of phenols is catalyzed by heterogeneous palladium on alumina and tolerates a variety of functional groups, giving access to building blocks with the opposite diastereoselectivity preferentially generated through the hydrogenation of arenes. The diastereoselectivity was inverted by employing rhodium-based catalysts and a simple and practical 
method for the chemoselective hydrogenation of phenols to cyclohexanones was established.

\section{ASSOCIATED CONTENT}

\section{SI Supporting Information}

The Supporting Information is available free of charge at https://pubs.acs.org/doi/10.1021/acscatal.0c03423.

Materials and methods, optimization of reaction condition, experimental procedure and characterization data for products, and synthesis procedure and characterization (PDF)

X-ray diffraction data (CIF)

\section{AUTHOR INFORMATION}

\section{Corresponding Author}

Frank Glorius - Organisch-Chemisches Institut, Westfälische Wilhelms-Universität Münster, 48149 Münster, Germany; ○ orcid.org/0000-0002-0648-956X; Email: glorius@unimuenster.de

\section{Authors}

Marco Wollenburg - Organisch-Chemisches Institut, Westfälische Wilhelms-Universität Münster, 48149 Münster, Germany

Arne Heusler - Organisch-Chemisches Institut, Westfälische Wilhelms-Universität Münster, 48149 Münster, Germany

Klaus Bergander - Organisch-Chemisches Institut, Westfälische Wilhelms-Universität Münster, 48149 Münster, Germany

Complete contact information is available at:

https://pubs.acs.org/10.1021/acscatal.0c03423

\section{Author Contributions \\ ¥(M.W., A.H.) These authors contributed equally. \\ Notes}

The authors declare no competing financial interest.

\section{ACKNOWLEDGMENTS}

Generous financial support by the Deutsche Forschungsgemeinschaft (IRTG 2027 Münster-Toronto) and the European Research Council (ERC Advanced Grant Agreement No. 788558) are gratefully acknowledged. The authors thank Daniel Moock, Tobias Wagener (both WWU Münster) and Austin D. Marchese (University of Toronto) for helpful discussions. We also thank Dr. Constantin G. Daniliuc (WWU Münster) for X-ray crystallographic analysis.

\section{REFERENCES}

(1) For reviews on arene hydrogenation, see: (a) Wiesenfeldt, M. P.; Nairoukh, Z.; Dalton, T.; Glorius, F. Selective Arene Hydrogenation for Direct Access to Saturated Carbo- and Heterocycles. Angew. Chem., Int. Ed. 2019, 58, 10460. (b) Giustra, Z. X.; Ishibashi, J. S. A.; Liu, S.-Y. Homogeneous Metal Catalysis for Conversion between Aromatic and Saturated Compounds. Coord. Chem. Rev. 2016, 314, 134. (c) Gualandi, A.; Savoia, D. Substrate Induced Diastereoselective Hydrogenation/Reduction of Arenes and Heteroarenes. RSC Adv. 2016, 6, 18419. (d) Qi, S.-C.; Wei, X.-Y.; Zong, Z.-M.; Wang, Y.-K. Application of Supported Metallic Catalysts in Catalytic Hydrogenation of Arenes. RSC Adv. 2013, 3, 14219. (e) Gual, A.; Godard, C.; Castillón, S.; Claver, C. Soluble Transition-Metal NanoparticlesCatalysed Hydrogenation of Arenes. Dalton Trans. 2010, 39, 11499. Soluble Transition-Metal Nanoparticles-Catalysed Hydrogenation of Arenes. For books on arene hydrogenation, see: (f) Foubelo, F.; Yus, M. Arene Chemistry: Reaction Mechanisms and Methods for Aromatic
Compounds; Mortier, J., Ed.; Wiley: Hoboken, 2016; p 337. (g) Bianchini, C.; Meli, A.; Vizza, F. The Handbook of Homogeneous Hydrogenation; de Vries, J. G.; Elsevier, C. J., Eds.; Wiley-VCH: Weinheim, 2006; p 455. (h) Nishimura, S. Handbook of Heterogeneous Catalytic Hydrogenation for Organic Synthesis; John Wiley \& Sons: New York, 2001; p 414. For selected reviews on the more general dearomatization of arenes, see: (i) Wertjes, W. C.; Southgate, E. H.; Sarlah, D. Recent Advances in Chemical Dearomatization of Nonactivated Arenes. Chem. Soc. Rev. 2018, 47, 7996. (j) Huck, C. J.; Sarlah, D. Shaping Molecular Landscapes: Recent Advances, Opportunities, and Challenges in Dearomatization. Chem. 2020, 6, 1589. (k) You, S.-L., Ed., Asymmetric Dearomatization Reactions; Wiley-VCH: Weinheim, 2016.

(2) Weissermel, K.; Arpe, H.-J. Industrial Organic Chemistry; WileyVCH: Weinheim, 2008; p 337.

(3) (a) Foppa, L.; Dupont, J. Benzene Partial Hydrogenation: Advances and Perspectives. Chem. Soc. Rev. 2015, 44, 1886. (b) Nagahara, H.; Ono, M.; Konishi, M.; Fukuoka, Y. Partial Hydrogenation of Benzene to Cyclohexene. Appl. Surf. Sci. 1997, $121-122,448$.

(4) (a) Wiesenfeldt, M. P.; Nairoukh, Z.; Li, W.; Glorius, F. Hydrogenation of Fluoroarenes: Direct Access to all-cis-(Multi)fluorinated Cycloalkanes. Science 2017, 357, 908. (b) Nairoukh, Z.; Wollenburg, M.; Schlepphorst, C.; Bergander, K.; Glorius, F. The Formation of all-cis-(Multi)fluorinated Piperidines by a Dearomatization-Hydrogenation Process. Nat. Chem. 2019, 11, 264. (c) Zhang, X.; Ling, L.; Luo, M.; Zeng, X. Accessing Difluoromethylated and Trifluoromethylated cis-Cycloalkanes and Saturated Heterocycles: Preferential Hydrogen Addition to the Substitution Sites for Dearomatization. Angew. Chem., Int. Ed. 2019, 58, 16785. (d) Wollenburg, M.; Moock, D.; Glorius, F. Hydrogenation of Borylated Arenes. Angew. Chem., Int. Ed. 2019, 58, 6549. (e) Ling, L.; He, Y.; Zhang, X.; Luo, M.; Zeng, X. Hydrogenation of (Hetero)aryl Boronate Esters with a Cyclic (Alkyl)(amino)carbene-Rhodium Complex: Direct Access to cis-Substituted Borylated Cycloalkanes and Saturated Heterocycles. Angew. Chem., Int. Ed. 2019, 58, 6554. (f) Wiesenfeldt, M. P.; Knecht, T.; Schlepphorst, C.; Glorius, F. Silylarene Hydrogenation: A Strategic Approach that Enables Direct Access to Versatile Silylated Saturated Carbo- and Heterocycles. Angew. Chem., Int. Ed. 2018, 57, 8297. (g) Wei, Y.; Rao, B.; Cong, X.; Zeng, X. Highly Selective Hydrogenation of Aromatic Ketones and Phenols Enabled by Cyclic (Amino)(alkyl)carbene Rhodium Complexes. J. Am. Chem. Soc. 2015, 137, 9250.

(5) For reviews on enantioselective hydrogenation of (hetero) arenes, see: (a) Zhao, D.; Candish, L.; Paul, D.; Glorius, F. NHeterocyclic Carbenes in Asymmetric Hydrogenation. ACS Catal. 2016, 6, 5978. (b) He, Y.-M.; Song, F.-T.; Fan, Q.-H. Advances in Transition Metal-Catalyzed Asymmetric Hydrogenation of Heteroaromatic Compounds. Top. Curr. Chem. 2013, 343, 145. (c) Wang, D.-S.; Chen, Q.-A.; Lu, S.-M.; Zhou, Y.-G. Asymmetric Hydrogenation of Heteroarenes and Arenes. Chem. Rev. 2012, 112, 2557. (d) Glorius, F. Asymmetric Hydrogenation of Aromatic Compounds. Org. Biomol. Chem. 2005, 3, 4171.

(6) Only few limited examples of trans-selective arene hydrogenation $($ trans $/$ cis $=>50 /<50)$ with scarce scope entries have been reported in the literature: (a) Murugesan, K.; Senthamarai, T.; Alshammari, A. S.; Altamimi, R. M.; Kreyenschulte, C.; Pohl, M.-M.; Lund, H.; Jagadeesh, R. V.; Beller, M. Cobalt-Nanoparticles Catalyzed Efficient and Selective Hydrogenation of Aromatic Hydrocarbons. ACS Catal. 2019, 9, 8581. (b) Li, H.; Wang, Y.; Lai, Z.; Huang, K.-W. Selective Catalytic Hydrogenation of Arenols by a Well-Defined Complex of Ruthenium and Phosphorus-Nitrogen $\mathrm{PN}^{3}$-Pincer Ligand Containing a Phenanthroline Backbone. ACS Catal. 2017, 7, 4446. (c) Tungler, A.; Szabados, E. Overcoming Problems at Elaboration and Scale-up of Liquid-Phase $\mathrm{Pd} / \mathrm{C}$ Mediated Catalytic Hydrogenations in Pharmaceutical Production. Org. Process Res. Dev. 2016, 20, 1246. (d) Maegawa, T.; Akashi, A.; Yaguchi, K.; Iwasaki, Y.; Shigetsura, M.; Monguchi, Y.; Sajiki, H. Efficient and Practical Arene Hydrogenation by Heterogeneous Catalysts under Mild Conditions. 
Chem. - Eur. J. 2009, 15, 6953. (e) Jansat, S.; Picurelli, D.; Pelzer, K.; Philippot, K.; Gómez, M.; Muller, G.; Lecante, P.; Chaudret, B. Synthesis, Characterization and Catalytic Reactivity of Ruthenium Nanoparticles Stabilized by Chiral N-Donor Ligands. New J. Chem. 2006, 30, 115. (f) Yadav, G. D.; Goel, P. K. Stereoselective Hydrogenation of $p$-tert-Butylphenol over Supported Rhodium Catalyst. J. Mol. Catal. A: Chem. 2002, 184, 281.

(7) (a) Van de Vyver, S.; Román-Leshkov, Y. Emerging Catalytic Processes for the Production of Adipic Acid. Catal. Sci. Technol. 2013, 3, 1465. (b) Musser, M. T. Cyclohexanol and Cyclohexanone. Ullmann's Encyclopedia of Industrial Chemistry; Wiley-VCH: Weinheim, 2011.

(8) See the Supporting Information for experimental details, full optimization data, and structural elucidation.

(9) Pitzer, L.; Schäfers, F.; Glorius, F. Rapid Assessment of the Reaction-Condition-Based Sensitivity of Chemical Transformations. Angew. Chem., Int. Ed. 2019, 58, 8572.

(10) (a) Bartholomew, C. H. Mechanisms of Catalyst Deactivation. Appl. Catal., A 2001, 212, 17. (b) Forzatti, P.; Lietti, L. Catalyst Deactivation. Catal. Today 1999, 52, 165.

(11) (a) Shu, R.; Li, R.; Lin, B.; Wang, C.; Cheng, Z.; Chen, Y. A Review on the Catalytic Hydrodeoxygenation of Lignin-Derived Phenolic Compounds and the Conversion of Raw Lignin to Hydrocarbon Liquid Fuels. Biomass Bioenergy 2020, 132, 105432. (b) Shafaghat, H.; Rezaei, P. S.; Ashri Wan Daud, W. M. Effective Parameters on Selective Catalytic Hydrodeoxygenation of Phenolic Compounds of Pyrolysis Bio-Oil to High-Value Hydrocarbons. RSC Adv. 2015, 5, 103999. (c) Saidi, M.; Samimi, F.; Karimipourfard, D.; Nimmanwudipong, T.; Gates, B. C.; Rahimpour, M. R. Upgrading of Lignin-Derived Bio-Oils by Catalytic Hydrodeoxygenation. Energy Environ. Sci. 2014, 7, 103. (d) Wang, D.-W.; Lu, S.-M.; Zhou, Y.-G. A Simple and Highly Effective Method for Hydrogenation of Arenes by $[\mathrm{Rh}(\mathrm{COD}) \mathrm{Cl}]_{2}$. Tetrahedron Lett. 2009, 50, 1282. (e) Hiyoshi, N.; Bando, K. K.; Sato, O.; Yamaguchi, A.; Rode, C. V.; Shirai, M. Stereoselective Hydrogenation of 4-Alkylphenols over CarbonSupported Rhodium Catalyst in Supercritical Carbon Dioxide Solvent. Catal. Commun. 2009, 10, 1702.

(12) For mechanistic analysis on the $\mathrm{Rh}(\mathrm{CAAC})$-catalyzed arene hydrogenation, see: (a) Moock, D.; Wiesenfeldt, M. P.; Freitag, M.; Muratsugu, S.; Ikemoto, S.; Knitsch, R.; Schneidewind, J.; Baumann, W.; Schäfer, A. H.; Timmer, A.; Tada, M.; Hansen, M. R.; Glorius, F. Mechanistic Understanding of the Heterogeneous, Rhodium-Cyclic (Alkyl)(Amino)Carbene-Catalyzed (Fluoro-)Arene Hydrogenation. ACS Catal. 2020, 10, 6309. (b) Tran, B. L.; Fulton, J. L.; Linehan, J. C.; Balasubramanian, M.; Lercher, J. A.; Bullock, R. M. Operando XAFS Studies on $\mathrm{Rh}(\mathrm{CAAC})$-Catalyzed Arene Hydrogenation. ACS Catal. 2019, 9, 4106. (c) Tran, B. L.; Fulton, J. L.; Linehan, J. C.; Lercher, J. A.; Bullock, R. M. Rh(CAAC)-Catalyzed Arene Hydrogenation: Evidence for Nanocatalysis and Sterically Controlled SiteSelective Hydrogenation. ACS Catal. 2018, 8, 8441.

(13) For selected references on the chemoselective hydrogenation of phenols to cyclohexanones, see: (a) Kong, X.; Gong, Y.; Mao, S.; Wang, Y. Selective Hydrogenation of Phenol. ChemNanoMat 2018, 4, 432. (b) Wei, Y.; Zeng, X. Catalytic Strategies toward Selective Hydrogenation of Aromatic Ketones and Phenols: Facile Synthesis of Cyclohexyl Ketones. Synlett 2016, 27, 650. (c) Zhong, J.; Chen, J.; Chen, L. Selective Hydrogenation of Phenol and Related Derivatives. Catal. Sci. Technol. 2014, 4, 3555. (d) Nelson, N. C.; Manzano, J. S.; Sadow, A. D.; Overbury, S. H.; Slowing, I. I. Selective Hydrogenation of Phenol Catalyzed by Palladium on High-Surface-Area Ceria at Room Temperature and Ambient Pressure. ACS Catal. 2015, 5, 2051. (e) Snelders, D. J. M.; Yan, N.; Gan, W.; Laurenczy, G.; Dyson, P. J. Tuning the Chemoselectivity of Rh Nanoparticle Catalysts by SiteSelective Poisoning with Phosphine Ligands: The Hydrogenation of Functionalized Aromatic Compounds. ACS Catal. 2012, 2, 201. (f) Wang, Y.; Yao, J.; Li, H.; Su, D.; Antonietti, M. Highly Selective Hydrogenation of Phenol and Derivatives over a Pd@Carbon Nitride Catalyst in Aqueous Media. J. Am. Chem. Soc. 2011, 133, 2362. (g) Liu, H.; Jiang, T.; Han, B.; Liang, S.; Zhou, Y. Selective Phenol
Hydrogenation to Cyclohexanone Over a Dual Supported Pd-Lewis Acid Catalyst. Science 2009, 326, 1250.

(14) (a) Hiyoshi, N.; Rode, C. V.; Sato, O.; Tetsuka, H.; Shirai, M. Stereoselective Hydrogenation of tert-Butylphenols over CharcoalSupported Rhodium Catalyst in Supercritical Carbon Dioxide Solvent. J. Catal. 2007, 252, 57. (b) Konuspaev, S. R.; Zhanbekov, K. N.; Kul'kova, N. V.; Murzin, D. Y. Kinetics of 4-tert-Butylphenol Hydrogenation over Rhodium. Chem. Eng. Technol. 1997, 20, 144. (c) Smith, H. A.; Stump, B. L. A Study of the Catalytic Hydrogenation of Hydroxybenzenes over Platinum and Rhodium Catalysts. J. Am. Chem. Soc. 1961, 83, 2739.

(15) (a) Weitkamp, A. W. Stereochemistry and Mechanism of Hydrogenation of Naphthalenes on Transition Metal Catalysts and Conformational Analysis of the Products. Adv. Catal. 1968, 18, 1. (b) Siegel, S.; Smith, G. V.; Dmuchovsky, B.; Dubbell, D.; Halpern, W. The Stereochemistry of the Hydrogenation of the Isomeric Xylenes and p-tert-Butyltoluene over a Platinum Catalyst. J. Am. Chem. Soc. 1962, 84, 3136.

(16) Brown, G.; Mangan, D.; Miskelly, I.; Moody, T. S. A Facile Stereoselective Biocatalytic Route to the Precursor of Woody Acetate. Org. Process Res. Dev. 2011, 15, 1036.

(17) (a) Weiser, T.; Wilson, N. Inhibition of Tetrodotoxin (TTX)Resistant and TTX-Sensitive Neuronal $\mathrm{Na}^{+}$Channels by the Secretolytic Ambroxol. Mol. Pharmacol. 2002, 62, 433. (b) Latli, B.; Hrapchak, M.; Switek, H.-K.; Retz, D. M.; Krishnamurthy, D.; Senanayake, C. H. Synthesis of Labeled Ambroxol and its Major Metabolites. J. Labelled Compd. Radiopharm. 2010, 53, 15. 